\title{
III Diretrizes para uso da Monitorização Ambulatorial da Pressão Arterial
}

\section{Diretriz para uso da Monitorização Residencial da Pressão Arterial}

\author{
7 a 9 de Setembro de 2000
}

\author{
Ilha de Comandatuba
}




\section{Participantes}

\author{
Andréa A. Brandão (RJ) \\ Angela Maria Geraldo Pierin (SP) \\ Celso Amodeo (SP) \\ Dante Marcelo Artigas Giorgi (SP) \\ Décio Mion Jr. (SP) \\ Fernando Nobre (SP)
}

\author{
Guido Rosito (RS) \\ Hilton Chaves Jr. (PE) \\ Istênio Fernandes Pascoal (DF) \\ João Cezar Mendes Moreira (SP) \\ José Luis Santello (SP)
}

\author{
José Márcio Ribeiro (MG) \\ Lilian Soares da Costa Mesquita(RJ) \\ Luis Aparecido Bortolotto (SP) \\ Marco Antonio Mota Gomes (AL) \\ Osvaldo Kohlmann Jr. (SP)
}

Nota: Texto mantido para publicação conforme original recebido pela Revista.

\author{
Paulo César Veiga Jardim (GO) \\ Raimundo Nascimento (MG) \\ Vera Koch (SP) \\ Wille Oigman (RJ)
}

\section{GRUPOVERMELHO}

\section{Moderador: José Márcio Ribeiro}

Secretário: Celso Amodeo

Componentes: Osvaldo Kohlmann, Lilian S. C. Mesquita, José Luis Santello, João Cezar Mendes Moreira

\section{Temática}

Comportamento fisiológico da pressão em $24 \mathrm{~h}$

Indicações, limitações e vantagens da monitorização ambulatorial da pressão arterial

Técnicas e métodos para obtenção da pressão arterial em $24 \mathrm{~h}$

Equipamentos: tipos, critérios de validação e equipamentos validados

Protocolos para realização do exame

Orientações indispensáveis ao paciente para realização da monitorização ambulatorial da pressão arterial

Cuidados na instalação do equipamento e cuidados para sua manutenção

\section{GRUPOVERDE}

Moderador: Dante Marcelo Giorgi

Secretário: Istênio Fernandes Pascoal

Componentes: Vera Koch, Guido Rosito, Hilton Chaves

\section{Temática}

Reprodutibilidade do método

Valores de normalidade e considerações, incluindo as limitações e populações especiais, como crianças e grávidas, dos dados obtidos com o exame

Conceito de efeito e hipertensão do avental branco

Significado clínico e prognóstico da hipertensão do avental branco

Importância do efeito do avental branco nos pacientes sob tratamento anti-hipertensivo

Papel da monitorização ambulatorial da pressão arterial na avaliação prognóstica dos pacientes hipertensos

\author{
Comissão de Redação \\ Dante Marcelo Artigas Giorgi \\ Décio Mion Jr. \\ Fernando Nobre \\ José Márcio Ribeiro
}

\section{GRUPOAZUL}

\author{
Moderador: Fernando Nobre \\ Secretário: Andréa Brandão \\ Componentes: Wille Oigman, Raimundo Nascimento, Paulo \\ César Jardim

\section{Temática} \\ Avaliação dos dados obtidos com a monitorização ambula- \\ torial da pressão arterial \\ Critérios de validade do exame \\ Definição, análise, critérios para interpretação de: pressões \\ sistólicas, pressões diastólicas, cargas pressóricas, picos \\ tensionais e episódios de hipotensão, variações da pressão \\ entre vigília e sono, variabilidade \\ Produção de relatórios
}

\section{GRUPOAMARELO}

\author{
Moderador: Décio Mion Jr. \\ Secretário: Angela Maria G. Pierin \\ Componentes:LuisBortolotto,Marco AntonioMotaGomes
}

\section{Temática}

Importância da monitorização residencial da pressão arterial Indicações, vantagens e limitações da monitorização residencial da pressão arterial

Equipamentos

Protocolos

Valor da monitorização residencial da pressão arterial para: diagnóstico de hipertensão arterial, avaliação da terapêutica, prognóstico do hipertenso

Critérios da normalidade

Interpretação dos dados obtidos com a monitorização residencial da pressão arterial e produção de relatórios

Correspondência: Fernando Nobre - Av. Bandeirantes, 3900 - 14049-900 Ribeirão Preto, SP - E-mail: fernando.nobre@uol.com.br 


\section{Apresentação}

A monitorização ambulatorial da pressão arterial tem apresentado um grande crescimento nos últimos anos, particularmente na última década, em que se concentra a maior parte das publicações que vieram definir aspectos de grande importância para a consolidação do método. Dois terços de todas as publicações, em periódicos indexados, concentramse nos últimos dez anos. Isso nos leva a uma revisão das últimas diretrizes publicadas em 1996.

Assim, a Sociedade Brasileira de Hipertensão e os Departamentos de Hipertensão das Sociedades Brasileiras de Cardiologia e Nefrologia reuniram-se à busca de normatizações, até onde os conhecimentos atualmente disponíveis permitem, para a utilização da monitorização ambulatorial da pressão arterial em nosso país.

Por outro lado, ganha consistência, particularmente nos últimos anos, a monitorização residencial da pressão arterial, que também e, pela primeira vez, merece atenção de um documento orientador à sua prática.

A reunião de um grupo de especialistas nessas áreas importantes e fundamentais para a melhor orientação do diagnóstico da hipertensão, para o estabelecimento do prognóstico do paciente hipertenso e para a melhor orientação da terapêutica anti-hipertensiva, utilizando os recursos que podem ser auferidos da monitorização ambulatorial da pressão arterial e da monitorização residencial da pressão arterial, representa uma contribuição necessária e oportuna. 


\section{Diretrizes para Uso da Monitorização Ambulatorial da Pressão Arterial - monitorização ambulatorial da pressão arterial}

\section{Comportamento fisiológico da pressão arte- rial nas $24 \mathrm{~h}$}

A pressão arterial varia devido à interação de fatores neuro-humorais, comportamentais e ambientais. A tabela I relaciona alguns desses principais fatores ${ }^{1}$. Observa-se uma variação contínua da pressão arterial de momento a momento, de acordo com as atividades do indivíduo, sendo que em pacientes hipertensos essa variabilidade da pressão arterial apresenta uma amplitude maior que nos indivíduos normais ${ }^{1}$. Durante o período de vigília, esses valores são maiores do que aqueles obtidos durante o sono. Em função dessas variações novos métodos surgiram para melhor avaliação do comportamento da pressão arterial em períodos predeterminados. Dentre eles destacam-se: medidas repetidas casuais da pressão arterial, monitorização ambulatorial e/ou residencial da pressão arterial.

\section{Indicações e limitações da monitorização ambulatorial da pressão arterial}

A monitorização ambulatorial da pressão arterial permite que seja realizado um grande número de medidas usualmente em $24 \mathrm{~h}$ - possibilitando o conhecimento do perfil de variações da pressão arterial na vigília e no sono. Entretanto, deve ser salientado que atualmente não existem evidências comprovadas de melhor avaliação de desfechos primários com a monitorização ambulatorial da pressão arterial do que com as medidas casuais da pressão arterial. A monitorização ambulatorial da pressão arterial é útil na avaliação de algumas situações clínicas, como expressa a tabela II. Por outro lado, algumas condições eventualmente presentes podem limitar a utilização da monitorização ambulatorial da pressão arterial. As principais limitações ao seu uso estão na tabela III.

\begin{tabular}{|lcc|}
\hline \multicolumn{3}{|c|}{ Tabela I - Variações da pressão arterial de acordo com diversas } \\
situações e atividades \\
\hline Atividades & $\begin{array}{c}\text { Pressão Sistólica } \\
(\mathrm{mmHg})\end{array}$ & $\begin{array}{c}\text { Pressão Diastólica } \\
(\mathrm{mmHg})\end{array}$ \\
\hline Reuniões & $+20,2$ & $+15,0$ \\
Trabalho & $+16,0$ & $+13,0$ \\
Caminhar & $+12,0$ & $+5,5$ \\
Vestir & $+11,5$ & $+9,7$ \\
Tarefas domésticas & $+10,7$ & $+6,7$ \\
Telefonar & $+9,5$ & $+7,2$ \\
Conversar & $+6,7$ & $+6,7$ \\
Assistir a TV & $+0,3$ & $+1,1$ \\
Repouso & 0 & 0 \\
Dormir & $-10,0$ & $-7,6$ \\
\hline
\end{tabular}

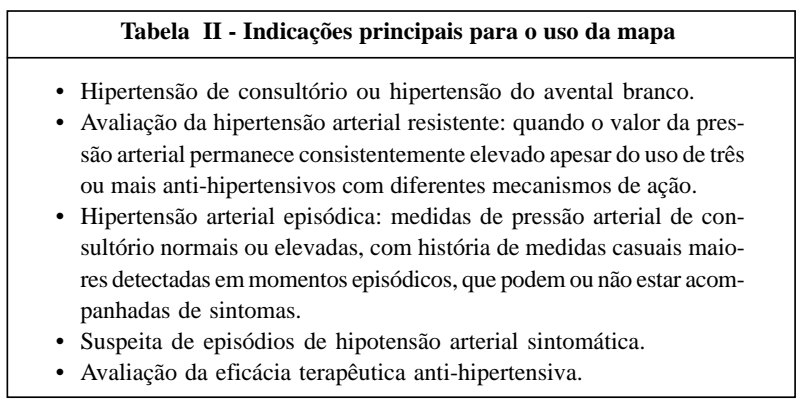

Tabela III - Principais limitações para o uso da monitorização ambulatorial da pressão arterial

- Arritmias cardíacas.

- Hipercinesia.

- Braços que não permitam o perfeito ajuste do manguito.

- Hiato auscultatório.

\section{Técnicas e métodos para obtenção da pressão arterial nas $24 \mathrm{~h}$}

Existem métodos diretos e indiretos de medida da pressão arterial. A monitorização ambulatorial da pressão arterial se baseia na medida indireta e intermitente da pressão arterial. As medidas indiretas podem ser obtidas pelo método auscultatório ou oscilométrico.

O método auscultatório baseia-se na detecção do primeiro e do último ruído de Korotkoff, enquanto o método oscilométrico, através da identificação do ponto de oscilação máxima, determina a pressão arterial média e as pressões sistólicas e diastólicas por meio de algoritmo específico $^{2}$. O mais utilizado atualmente é o método oscilométrico.

\section{Equipamentos}

Para a utilização dos aparelhos de monitorização ambulatorial da pressão arterial disponíveis no mercado devem ser levados em consideração, no momento da compra, os aspectos citados na tabela IV.

\section{Protocolo para realização do exame ${ }^{3}$}

Para realização da monitorização ambulatorial da pressão arterial são necessárias as orientações e cuidados explicitados na tabela $\mathrm{V}$. 
Tabela IV - Fatores a serem considerados na aquisição de um aparelho de monitorização ambulatorial da pressão arterial $^{3}$

- Aparelho validado pela British Hypertension Society e/ou pela US Association for the Advancement of Medical Instrumentation.

- Custo do aparelho.

- Custo do software.

- Informações contidas no software.

- Instruções adequadas do manual.

- Custo de manutenção.

- Custo do material de consumo (ex.: baterias, manguitos etc.).

- Assistência técnica disponível.

- Garantia.

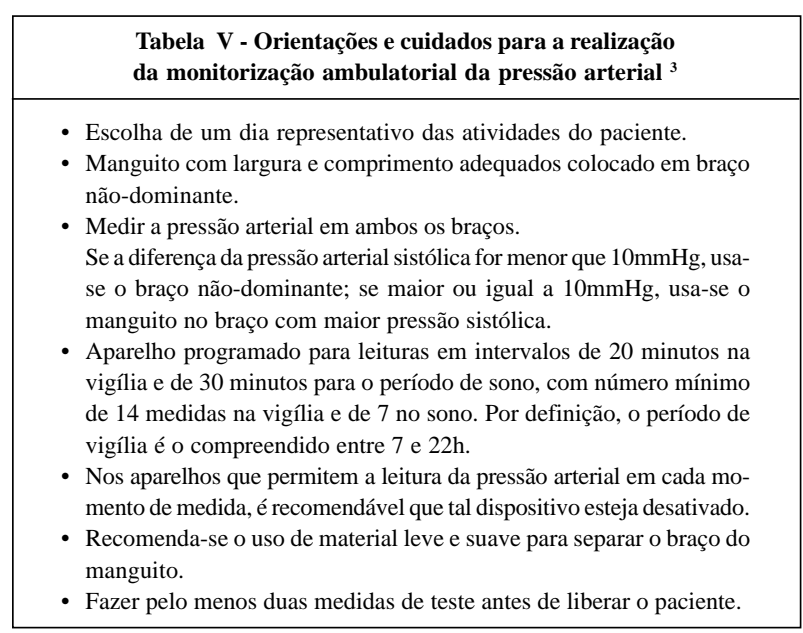

\section{Explicações adequadas do procedimento}

O paciente que será submetido ao exame deverá receber orientações mínimas indispensáveis no sentido de obtenção de procedimento de boa qualidade. Essas orientações básicas estão listadas na tabela VI.

\section{Cuidados para manutenção e calibração dos aparelhos}

Deve-se exigir de cada fabricante as recomendações específicas de manutenção e calibração necessárias a cada monitor.

Esforços serão desenvolvidos pelas Sociedades Bra- sileiras de Cardiologia, Hipertensão e Nefrologia em busca de ações junto aos organismos oficiais controladores de qualidade e calibração de equipamentos, como o INMETRO, para a criação de recomendações nesse sentido.

\section{Reprodutibilidade do método}

Para a população de indivíduos normotensos e hipertensos, independentemente do sexo ou da idade, a monitorização ambulatorial da pressão arterial é um exame que apresenta boa reprodutibilidade. Os valores da pressão arterial sistólica, diastólica e média, bem como a frequiência cardíaca obtida em $24 \mathrm{~h}$, vigília e sono, apresentam resultados semelhantes em exames consecutivos realizados em curto intervalo de tempo ${ }^{4,5}$.

A maioria dos pacientes apresenta diminuição da pressão arterial durante o sono. A variação da pressão arterial entre os períodos de sono e vigília também apresenta boa reprodutibilidade quando considerada como variável contínua ${ }^{4}$. Entretanto, a variação da pressão arterial entre os períodos de vigília e sono tem sido considerada em duas categorias distintas. Quando ocorre queda da pressão arterial igual ou superior a $10 \%$ em relação à pressão de vigília, define-se o indivíduo como com queda da pressão arterial durante o sono ou, em inglês, como dipper. Quando a queda é inferior a $10 \%$, é caracterizado como sem queda da pressão arterial durante o sono ou, em inglês, como non-dipper. A reprodutibilidade do caráter dipper e non-dipper tem sido questionada na literatura devido à chance de $30 \%$ a $50 \%$ dos indivíduos mudarem de categoria em exames subseqüentes ${ }^{6}$. Essa aparente controvérsia se deve às limitações advindas da utilização de pontos de cortes arbitrários, nesta condição 10\%, para representar variáveis contínuas. Assim sendo, o fenômeno da variação da pressão arterial entre a vigília e o sono deve ser considerado em seu valor absoluto (em $\mathrm{mmHg}$ ) e percentual.

\section{Valores de normalidade para as pressões obtidas com a monitorização ambulatorial da pressão arterial}

À semelhança da medida casual da pressão arterial, os critérios de normalidade dos valores de pressão na monitorização ambulatorial da pressão arterial são arbitrários. A

\begin{tabular}{|c|c|c|c|}
\hline \multicolumn{4}{|c|}{$\begin{array}{c}\text { Tabela VII - Valores de normalidade para a monitorização } \\
\text { ambulatorial da pressão arterial }\end{array}$} \\
\hline Média da pressão & Normal & Intermediário & Anormal \\
\hline \multicolumn{4}{|l|}{ Sistólica } \\
\hline Vigília & $<135$ & $135-140$ & $>140$ \\
\hline Sono & $<120$ & $120-125$ & $>125$ \\
\hline $24 \mathrm{~h}$ & $<130$ & $130-135$ & $>135$ \\
\hline \multicolumn{4}{|l|}{ Diastólica } \\
\hline Vigília & $<85$ & $85-90$ & $>90$ \\
\hline Sono & $<75$ & $75-80$ & $>80$ \\
\hline $24 \mathrm{~h}$ & $<80$ & $80-85$ & $>85$ \\
\hline
\end{tabular}


tabela VII expressa os valores considerados normais, intermediários e anormais para a monitorização ambulatorial da pressão arterial. Para a população adulta, as medidas normais e anormais disponíveis e recomendadas representam apenas um guia para a interpretação do exame. Níveis mais baixos de pressão podem ter relevância clínica em pacientes com múltiplos fatores de risco ${ }^{7}$. É importante salientar que não existem estudos longitudinais com o uso da monitorização ambulatorial da pressão arterial na população brasileira.

As médias sistólicas e diastólicas na vigília apresentam geralmente valores mais elevados quando comparados aos obtidos por medida casual, enquanto as médias pressóricas sistólicas e diastólicas durante o sono mostram valores inferiores àqueles obtidos casualmente.

\section{Crianças}

Dados de monitorização ambulatorial da pressão arterial em crianças têm demonstrado boa correlação com idade cronológica, altura, peso e frequiência cardíaca, além de boa reprodutibilidade ${ }^{8}$.

Belsha e cols. ${ }^{9}$ compararam, através da monitorização ambulatorial da pressão arterial, o perfil de pressão arterial de vigília e sono de crianças e adolescentes normotensos e hipertensos, demonstrando que os pacientes hipertensos apresentam médias pressóricas mais elevadas, com perfil pressórico de $24 \mathrm{~h}$ semelhante aos seus pares normotensos. Não existem critérios estabelecidos de normalidade para as variáveis obtidas. Para cálculo das cargas pressóricas de vigília utilizam-se como limite superior de normalidade o percentil 95 para sexo e idade da curva de referência da pressão arterial casual, e valores $10 \%$ menores para as pressões de sono.

Contudo, a avaliação pela monitorização ambulatorial da pressão arterial de crianças e adolescentes com doenças de base com risco associado de desenvolvimento de hipertensão arterial tem sido descrita como promissora em pacientes com diabete melito tipo $1^{10}$, doença policística autossômica dominante ${ }^{11}$, na insuficiência renal crônica dialítica ${ }^{12,13}$ e em pacientes transplantados renais ${ }^{14}$. Nessas situações clínicas a monitorização ambulatorial da pressão arterial tem se mostrado mais útil para o diagnóstico e manejo da hipertensão arterial do que a pressão arterial de consultório, por fornecer dados sobre alterações do perfil pressórico durante o sono, reclassificar pacientes quanto ao diagnóstico de hipertensão arterial obtido pela medida em consultório e apresentar maior correlação com o desenvolvimento de lesões de órgãos-alvo.

\section{Grávidas}

A maioria dos estudos de parâmetros circadianos da pressão arterial durante a gravidez demonstra a preservação do padrão vigília-sono e a variabilidade global semelhante àquela observada em mulheres não-grávidas ${ }^{15,16}$. Igualmente, observa-se diminuição linear da pressão arterial sistólica, diastólica e média até a 22aㅗ semana de gestação, seguida por aumento progressivo até o dia do parto ${ }^{17-19}$.

Variações da pressão arterial na pré-eclâmpsia têm sido reconhecidas há muito tempo. Entretanto, a atenuação ou inversão do padrão vigília-sono, inicialmente descrita, apresenta baixa sensibilidade. Isto é, muitos casos de préeclâmpsia, definidos sob critério clínico ${ }^{20}$ ou histológico renal $^{21}$, não apresentam as alterações mencionadas relativas ao ritmo circadiano.

A possibilidade de antecipar o desenvolvimento de pré-eclâmpsia representaria o maior benefício que a monitorização ambulatorial da pressão arterial poderia trazer à propedêutica na mulher gestante. Níveis de pressão arterial média superiores a 95mmHg e de pressão arterial diastólica superiores a $80 \mathrm{mmHg}$ no primeiro trimestre e acima de $90 \mathrm{mmHge}$ $75 \mathrm{mmHg}$ respectivamente no segundo trimestre, embora dentro da faixa normal, foram inicialmente identificados como preditivos do desenvolvimento de pré-eclâmpsia ${ }^{22}$, po-

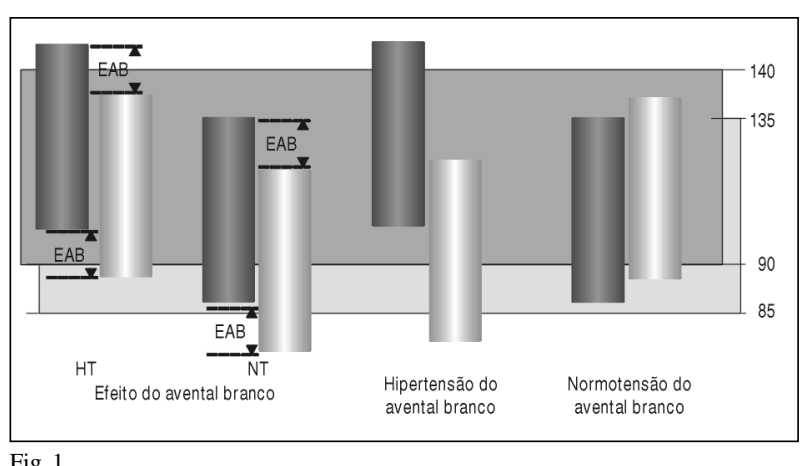

Fig. 1

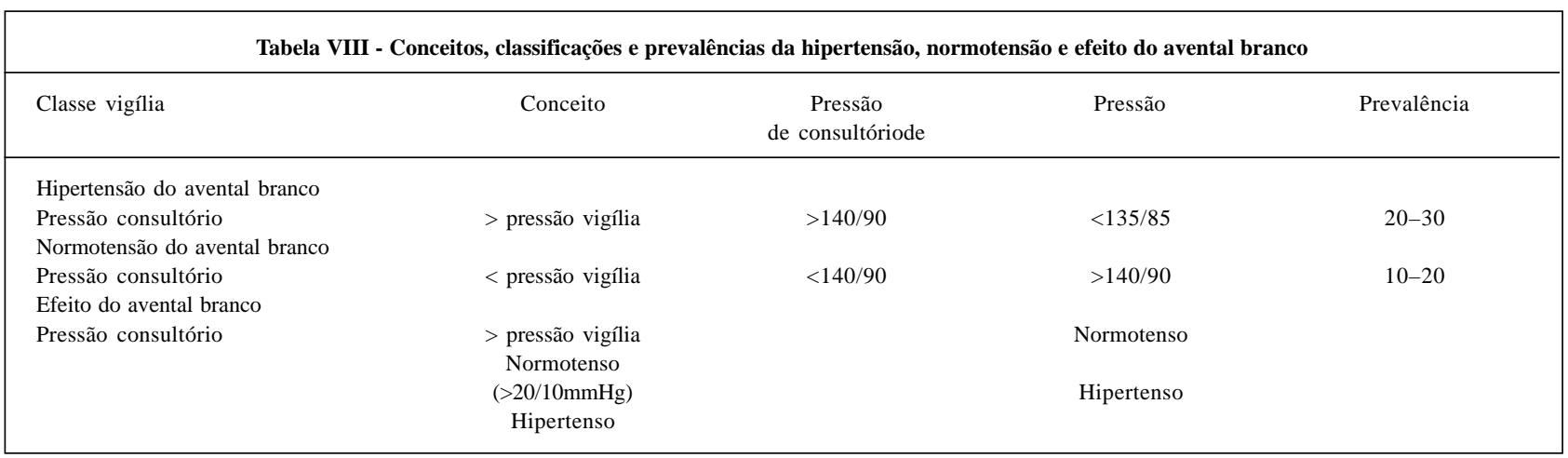


rém esses dados não foram convincentemente confirmados, em boa medida devido à ampla sobreposição de valores de pressão entre as pacientes que efetivamente desenvolvem pré-eclâmpsia e as que não desenvolvem ${ }^{23}$.

\section{Fenômeno do avental branco}

Os níveis da pressão arterial medidos em consultório podem ser maiores, semelhantes ou menores do que os obtidos em vigília pela monitorização ambulatorial da pressão arterial. Essas diferenças eventuais possibilitam a classificação dos pacientes em duas diferentes categorias: hipertensão do avental branco ${ }^{24}$ ou normotensão do avental branco $^{25}$ (fig. 1).

Registra-se igualmente o efeito do avental branco ${ }^{24}$. Conforme definições e valores expressos na tabela VIII, define-se cada situação específica.

Há evidências de que a hipertensão do avental branco possa não ser tão benigna como se pensava, ocorrendo maior possibilidade de acometimento de órgãos-alvo que em populações normotensas, porém em menor escala do que em hipertensos ${ }^{26}$. Por outro lado, alguns autores demonstram semelhante possibilidade de eventos cardiovasculares para este grupo de indivíduos quando comparados com uma população de normotensos ${ }^{27}$.

Não há características clínicas específicas que auxiliem neste diagnóstico, embora sua prevalência aumente com a idade, notadamente no sexo feminino. Não existem evidências de benefícios de intervenções medicamentosas neste grupo de pacientes; entretanto, o paciente deve ser considerado no contexto de seu risco cardiovascular global, permanecendo em seguimento clínico.

\section{Significado clínico e prognóstico da normoten- são do avental branco}

Recentemente, destacou-se um grupo de pacientes cuja pressão arterial é elevada pela monitorização ambulatorial da pressão arterial, mas apresenta valores normais na medida casual.

Estudos restritos têm sugerido que esses pacientes têm maior prevalência de lesões de órgãos-alvo do que indivíduos normotensos nos dois métodos de medida da pressão arterial $^{25,29}$.

\section{Papel da monitorização ambulatorial da pres- são arterial na avaliação prognóstica em paci- entes hipertensos}

Por serem mais reprodutíveis e melhor estimar a variável pressão arterial, os valores de pressão arterial obtidos pela monitorização ambulatorial da pressão arterial se correlacionam mais fortemente com lesões de órgãos-alvo e morbidade e mortalidade do que as medidas casuais da pressão arterial.

Os valores médios de pressão arterial sistólica e diastólica de $24 \mathrm{~h}$, de vigília e de sono apresentam correlação posi- tiva com índice de massa de ventrículo esquerdo, lesões isquêmicas encefálicas e microalbuminúria. Emidosos, avaliados no Estudo Syst-Eur, a variável que apresentou melhor correlação com eventos cardiovasculares maiores, como acidente vascular encefálico, infarto agudo do miocárdio e óbito foi a pressão arterial sistólica do sono, seguida da pressão arterial sistólica de 24 h e da pressão arterial sistólica de vigília. O estudo de Ohasama ${ }^{28}$ encontrou achados semelhantes.

A intensidade da queda da pressão arterial no sono mostra correlação inversa com a massa de ventrículo esquerdo e microalbuminúria. Em relação a lesões isquêmicas encefálicas, os indivíduos com atenuação da queda no sono $(<10 \%)$ ou com queda noturna exagerada (>20\%) apresentam maior número de áreas isquêmicas à ressonância nuclear magnética cerebral.

A variabilidade da pressão arterial estimada pelo desvio padrão (coeficiente de variação percentual) de medidas contínuas da pressão arterial batimento a batimento apresenta correlação positiva com lesões de órgãos-alvo. Entretanto, por possível limitação da monitorização ambulatorial da pressão arterial, essa associação não foi demonstrada com o desvio padrão (coeficiente de variação percentual) obtido pela monitorização ambulatorial da pressão arterial de $24 \mathrm{~h}$.

\section{Avaliação dos dados obtidos com a monitoriza- ção ambulatorial da pressão arterial}

Para a análise dos dados obtidos com a monitorização ambulatorial da pressão arterial há necessidade inicial de se avaliar a qualidade do exame. Os dados obtidos e avaliáveis são: médias pressóricas; diferenças de pressão vigíliasono; correlações entre pressões e atividades, sintomas e medicamentos; presença de picos tensionais e episódios de hipotensão. Outros parâmetros são obtidos, mas, ou não existem critérios definitivos para a sua interpretação, como pressão arterial média, pressão de pulso e variabilidade, ou são mal estimados pelos equipamentos disponíveis, tais como frequiência cardíaca e, finalmente, aqueles que têm im-

Tabela IX - Avaliação dos dados obtidos com a monitorização ambulatorial da pressão arterial

- Dados avaliáveis

- médias pressóricas

- diferenças vigília/sono

- relações pressão-atividade/sintomas/medicamentos

- picos tensionais

- hipotensão

- Sem critérios definidos para interpretação

- pressão arterial média

- pressão de pulso

- variabilidade

- Mal estimados pelos equipamentos - frequiência cardíaca

- Importância clínica limitada - cargas pressóricas 
portância clínica limitada, como, por exemplo, a carga pressórica (tab. IX).

\section{Validade do procedimento}

Thijs e cols. ${ }^{30}$ analisaram a validade do exame baseado no número mínimo de medidas necessárias em $24 \mathrm{~h}$ e concluíram que esse número mínimo deveria ser de, pelo menos, uma medida a cada 30 minutos. Considerando-se uma perda média de $20 \%$, recomenda-se a realização das medidas, no mínimo, a cada 20 minutos durante a vigília e a cada 30 minutos no período de sono. A exigência mínima é de pelo menos 14 medidas na vigília e 7 durante o sono. Em determinadas situações, tal como perda de medidas em horários não-relevantes, a juízo clínico, um número de medidas abaixo do preconizado pode ser aceitável.

\section{Médias de pressão arterial}

Dentre os parâmetros obtidos pela monitorização ambulatorial da pressão arterial, as médias de pressão arterial são os melhores dados a serem analisados, por apresentarem maiores índices de correlação com diagnóstico, lesão em órgãos-alvo e prognóstico cardiovascular, tendo sido o único parâmetro relacionado à mortalidade, de acordo com o estudo de Ohkubo e cols. ${ }^{28}$. A análise dos períodos de $24 \mathrm{~h}$, vigília e sono é considerada essencial para avaliação das médias de pressão. Períodos específicos, tais como as primeiras horas após o despertar ou na sesta, não têm ainda sua importância clínica bem estabelecida. Recomenda-se que esse parâmetro seja interpretado de acordo com os valores expressos na tabela VII, destacando-se a normalidade ou não dos valores obtidos. Deve-se alertar para a presença de médias consideradas intermediárias. Tanto as médias sistólicas quanto diastólicas, isoladas ou simultaneamente, têm valor clínico.

\section{Diferenças de pressão vigília-sono}

É fundamental para a definição dos períodos de vigília e de sono a anotação precisa dos horários em que o indivíduo submetido ao exame dormiu e acordou. Esses dados devem estar claramente anotados no diário de atividades.

A referência, pelo paciente, de sono intensamente perturbado na vigência do exame deve ser considerada no momento da interpretação das variações das pressões vigília-sono.

Usualmente encontra-se queda da pressão sistólica e diastólica durante o sono quando comparado ao período de vigília. Foi observado em hipertensos que queda inferior a $10 \%$ relacionava-se a pior prognóstico cardiovascular ${ }^{31}$. Em indivíduos normotensos, a ausência de queda da pressão arterial durante o sono não tem significado clínico confirmado. Shimada e cols. ${ }^{32}$ demonstraram, em idosos hipertensos com descenso superior a $20 \%$, que houve aumento de risco cardiovascular, especialmente para a ocorrência de acidente vascular encefálico.
Vale lembrar que a inversão do comportamento fisiológico da pressão vigília-sono ou ausência de queda podem estar relacionadas a determinadas situações, tais como distúrbio do sono provocado pelo exame, controle inadequado da pressão em pacientes tratados, em algumas situações, de hipertensão secundária, apnéia do sono, disautonomia e uso de algumas drogas, como a ciclosporina.

\section{Correlações de pressões com atividades, sinto- mas e medicamentos}

Para a devida análise desses itens, é essencial o preenchimento detalhado do diário de atividades, sendo de grande importância a anotação dos horários em que o paciente dormiu e acordou, usou medicamentos ou bebidas alcoólicas, café e tabaco.

A presença de sintomas, horário de trabalho ou atividade física também precisam ser detalhadamente descritos ${ }^{33}$.

\section{Picos hipertensivos e episódios de hipotensão}

Elevações significativas da pressão arterial de forma progressiva, em pelo menos duas medidas, atingindo um pico claramente acima das variações anterior e posterior configuram pico hipertensivo. Vale a pena ressaltar que valores elevados e isolados, na maioria das vezes, representam artefatos, portanto, sem significado.

Episódios sintomáticos de diminuição da pressão arterial podem ser importantes, especialmente nas seguintes situações: ação medicamentosa, síncope, lipotímia pósprandial em idosos, hipotensão postural, disautonomia e diabete melito, entre outros. Medidas isoladas e assintomáticas de diminuição acentuada da pressão arterial também podem ser decorrentes de artefatos técnicos.

\section{Pressão arterial média, pressão de pulso e va- riabilidade}

A pressão arterial média é obtida pelo exame, com aplicabilidade restrita à pesquisa clínica.

A despeito da grande importância clínica que se tem dado à pressão de pulso, com base nas medidas casuais, com fortes evidências de implicações prognósticas, não há até o momento critérios para a interpretação desse parâmetro pela monitorização ambulatorial da pressão arterial.

Sabe-se que a variabilidade da pressão arterial tem grande correlação prognóstica com eventos cardiovasculares e desenvolvimento de lesão em órgãos-alvo. Entretanto, a avaliação apropriada da variabilidade é somente possível através de registro contínuo da pressão arterial (batimento a batimento), o que não é obtido pelo método usual de registro da pressão arterial em $24 \mathrm{~h}$. O desvio padrão das médias de pressão arterial obtido pela monitorização ambulatorial da pressão arterial vem sendo inadequadamente utilizado como indicativo da variabilidade da pressão arterial. Entretanto, até o momento não há critérios de normalidade para sua interpretação. 
Tabela X - Itens que necessariamente deverão ser avaliados em um relatório de monitorização ambulatorial da pressão arterial

- Qualidade do exame

- Médias de pressão

- Diferenças de pressão vigília-sono

- Correlações entre pressões e atividades, sintomas e medicamentos

- Picos tensionais e episódios de hipotensão

- Comentários

- Conclusões

\section{Freqüência cardíaca}

Apesar de registrarem a freqüência cardíaca, os equipamentos utilizados para a monitorização ambulatorial da pressão arterial não são apropriados para a obtenção desse parâmetro, não devendo, portanto, ser considerado, exceto nos equipamentos capazes de registrar simultaneamente $\mathrm{o}$ eletrocardiograma em $24 \mathrm{~h}$.

\section{Cargas pressóricas}

Embora tenha definição proposta desde $1988^{34}$ - percentagens de medidas acima dos valores de referência -, este critério suporta várias críticas.

Dentre elas, uma das mais consistentes está relacionada ao fato de que o mesmo valor de cargas pressóricas pode significar diferentes comportamentos estimados pelas médias de pressão.

Mesmo havendo documentação sobre a relação direta entre valores de cargas, especialmente acima de $50 \%$, e lesões em órgãos-alvo, há a tendência, nas mais recentes diretrizes para utilização da monitorização ambulatorial da pressão arterial, de não se considerarem na interpretação clínica os valores de cargas pressóricas ${ }^{36-38}$.

Assim, pode-se julgar igualmente inapropriada a consideração dessa variável na interpretação rotineira do exame.

\section{Produção de relatórios}

O relatório da monitorização ambulatorial da pressão arterial deve conter obrigatoriamente os itens apontados na tabela $\mathrm{X}^{39}$.

Não se deve estabelecer, em conformidade com as informações hoje disponíveis, diagnóstico de hipertensão utilizando-se a monitorização ambulatorial da pressão arterial, pois este é um diagnóstico clínico. Quando indicada para fins de avaliação do comportamento da pressão em $24 \mathrm{~h}$, deve-se apenas considerar nas conclusões: comportamento normal, intermediário ou anormal da pressão em $24 \mathrm{~h}$. Para fins de avaliação da eficácia terapêutica deve-se concluir pelo seguinte: as medicações referidas como utilizadas parecem (ou não) estar exercendo adequado controle da pressão arterial nas $24 \mathrm{~h}$. 


\section{Diretrizes para o Uso da Monitorização Residencial da Pressão Arterial}

\section{Importância da monitorização residencial da pressão arterial}

A medida da pressão arterial no consultório, apesar de considerada procedimento padrão para o diagnóstico de hipertensão e para o seguimento de pacientes hipertensos, está sujeita a inúmeros fatores de erro, sendo que o mais importante dentre eles é a influência do observador. Além disso, tal medida propicia um número reduzido de leituras, que não apresentam boa reprodutibilidade ao longo do tempo.

A medida residencial realizada durante vários dias pelo paciente ou familiar devidamente treinados constitui uma alternativa útil para evitar esses inconvenientes. Essa nãoé uma técnica inovadora, pois já em $1940{ }^{40}$ havia sido demonstrado que a medida residencial, apresentava valores de 30 a 40mmHg mais baixos do que a medida no consultório. Comparando-se os valores de medida da pressão no consultório com a monitorização ambulatorial da pressão arterial e a medida residencial verificou-se que a residencial apresentou valores mais baixos do que a monitorização ambulatorial da pressão arterial e a pressão de consultório ${ }^{41}$.

Com o desenvolvimento de equipamentos compactos, confiáveis, validados e de preços mais acessíveis, o procedimento se tornou viável para uso em larga escala na prática clínica diária e na pesquisa.

Tabela XI - Vantagens da monitorização residencial da pressão arterial realizada com aparelho semi-automático em comparação às medidas de consultório

- Oferece maior número de medidas ${ }^{42}$

- Apresenta boa aceitabilidade inclusive por idosos e muito idosos ${ }^{43}$

- Favorece maior adesão ao tratamento ${ }^{44}$

- Mostra boa reprodutibilidade ${ }^{45}$

- Avalia a pressão sem influência do observador e do ambiente do consultório ${ }^{46}$

- Atenua os erros e preferências do observador ${ }^{47}$

- Apresenta menor efeito placebo ${ }^{48}$

- Mostra melhor correlação com lesão de órgãos-alvo ${ }^{46}$

- Possibilita armazenamento, impressão e transmissão a distância das leituras obtidas ${ }^{44}$

- Diminui o número de visitas ${ }^{49}$

- Os aparelhos têm menor custo ${ }^{50}$
Tabela XII - indicações da monitorização residencial da pressão arterial realizada com aparelho semi ou automático ${ }^{47,51,52}$

- Seguimento do hipertenso do avental branco

- Quantificação do efeito do avental branco

- Avaliação da eficácia terapêutica anti-hipertensiva

- Utilização em ensaios clínicos

\section{Indicações, vantagens e limitações da monitori- zação residencial da pressão arterial}

Dentre as vantagens da monitorização residencial da pressão arterial em relação às medidas casuais (de consultório) está a melhoria dos índices de adesão ao tratamento, o que levou vários organismos internacionais a sugerirem o uso rotineiro de tal procedimento.

\section{Equipamentos}

A medida da pressão arterial pelo paciente ou familiar pode ser realizada através de: esfigmomanômetro de coluna de mercúrio; esfigmomanômetro aneróide; aparelhos oscilométricos semi ou automáticos com deflagração manual de medida no braço, no pulso ou no dedo ${ }^{53}$.

O esfigmomanômetro de coluna de mercúrio não é apropriado para medida pelo paciente ou familiar devido às dificuldades do método indireto de medida com técnica auscultatória, além de o mercúrio estar sendo gradativamente banido do uso médico devido a sua toxicidade ${ }^{54}$. $\mathrm{O}$ aneróide apresenta as mesmas dificuldades do método indireto com técnica auscultatória, além da descalibração mais freqüente do que o de coluna de mercúrio.

Com relação aos aparelhos oscilométricos semi ou automáticos com deflagração manual, os de medida no braço são os mais recomendados e confiáveis. Os que medem a pressão no pulso apresentam limitações devido à necessidade de colocação do pulso ao nível do coração, além de erros devido à flexão ou hiperextensão do pulso durante a medida. Aqueles que medem no dedo, apesar de convenientes para o paciente, não são recomendáveis devido à baixa confiabilidade ${ }^{55}$. É fundamental que os aparelhos empregados sejam validados de acordo com as normas da British Hypertension Society e Association for Advancement of Medical Instruments ou outros critérios aceitos internacionalmente ${ }^{56}$. Além disso, é importante que o equipamento tenha sua calibração testada contra aparelho de coluna de mercúrio, pelo menos, anualmente.

Tabela XIII - Limitações da monitorização residencial da pressão arterial realizada com aparelho semi ou automático ${ }^{47,51,52}$

- Impossibilidade de medir a pressão durante o sono

- Tempo despendido na instrução do paciente e/ou familiares

- Número reduzido de estudos de normalidade e prognóstico

- Grande número de equipamentos não-validados

- Pacientes arrítmicos, obesos e crianças

- Possibilidade de o paciente auto-ajustar a medicação induzido pelo valor da leitura 
Outro aspecto relacionado ao equipamento diz respeito à necessidade do uso de manguito de tamanho adequado ao braço do paciente.

\section{Protocolos, procedimentos e instruções ao paciente}

Vários protocolos de monitorização residencial da pressão arterial têm sido utilizados ${ }^{53,57}$. Recomenda-se, pelo menos, duas medidas pela manhã, antes da tomada das medicações e do desjejum, e duas à noite, antes do jantar ou três horas depois, para evitar a redução pós-prandial da pressão arterial, durante, no mínimo, três dias consecutivos de atividades habituais, desprezando-se as medidas do primeirodia.

\section{Valor clínico da monitorização residencial da pressão arterial}

Diagnóstico da hipertensão do avental branco - A medida residencial da pressão arterial, por ser isenta do efeito do observador, possibilita o diagnóstico do efeito e da hipertensão do avental branco, mais freqüentemente observados nas hipertensões leves. No entanto, Stergiou e cols. ${ }^{58}$ mostraram que a medida residencial não foi apropriada como alternativa à monitorização ambulatorial da pressão arterial no diagnóstico da hipertensão do avental branco, podendo, porém, ser útil como método de rastreamento desse fenômeno e de acompanhamento a longo prazo dos hipertensos nessas condições devido a sua alta especificidade e baixo custo.

Avaliaçãoda eficácia da terapêutica anti-hipertensiva - Graças ao baixo custo ${ }^{50}$, boa aceitabilidade ${ }^{59}$, facilidade de manuseio, possibilidade de avaliação a longo prazo e monitorização a distância ${ }^{60}$, a medida residencial pode ser bastante apropriada para avaliação da eficácia da terapêutica anti-hipertensiva. Além disso, pode ser utilizada na avaliação do efeito de drogas anti-hipertensivas em ensaios clínicos, diminuindo o número de pacientes necessários para o estudo ${ }^{61}$.

A medida residencial é particularmente útil na avaliação da eficácia da terapêutica em pacientes hipertensos resistentes às medidas de consultório, por poder observar o efeito do avental branco ${ }^{62}$.

Tabela XV - Instruções gerais a serem fornecidas ao paciente para medida da pressão arterial no braço

- Informar sobre a variação da pressão arterial: “A pressão varia a cada batimento do coração"

- Salientar que, na maioria das pessoas, a pressão arterial em casa é mais baixa do que no consultório

- Informar que pressões com diferencial pequeno (140/130) geralmente são artefatos

- Orientar para a realização de medidas nos dias e horários recomendados pelo médico, sem alterar a rotina de vida

- Recomendar não medir a pressão arterial de outras pessoas com equipamento que armazena leituras

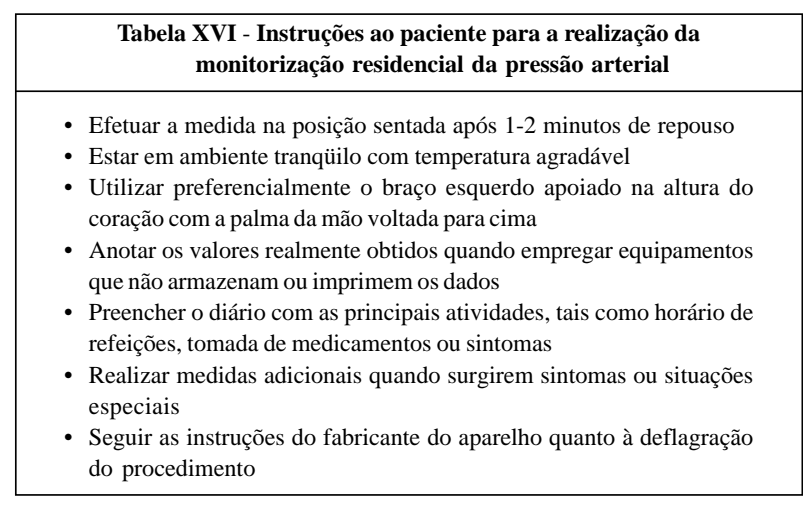

Prognóstico do hipertenso - Dois estudos prospectivos importantes avaliaram o papel prognóstico da medida residencial. Assim, o estudo de Ohasama ${ }^{62}$ analisou grande número de indivíduos durante vários anos, tendo observado mortes como desfecho, e demonstrou que a medida residencial apresenta correlação com mortalidade cardiovascular total, morbidade por acidente vascular encefálico e mortalidade não-cardiovascular. Ao comparar a medida residencial com a casual de consultório, os autores verificaram que aquela mostrou poder preditivo mais forte do que esta.

No estudo "Tecumseh" ${ }^{63}$ foi sugerido que a medida residencial pode ter valor preditivo para o desenvolvimento de hipertensão e disfunção diastólica do ventrículo esquerdo.

Vários estudos têm mostrado melhor correlação da medida residencial com lesões de órgãos-alvo. Em nosso meio foi demonstrada melhor correlação da medida residencial com índice de massa de ventrículo esquerdo quando se compara com a medida de consultório ${ }^{41}$.

\section{Critérios de normalidade}

Análise de banco de dados internacional, considerando medidas de manhã e noite, mostrou valores de normalidade menores ou iguais a 135/85mmHg e anormais acima de 140/ $90 \mathrm{mmHg}$, havendo zona de incerteza entre esses limites ${ }^{64}$.

Outro critério sugerido é empregar valores de medida residencial correspondentes a valores de medida de consultório. Assim, o valor correspondente a 130/85 $\mathrm{mmHg}$ no consultório seria $125 / 80 \mathrm{mmHg}$, e 140/90mmHg no consultório corresponderia a $133 / 86 \mathrm{mmHg}$ na monitorização residencial da pressão arterial ${ }^{65}$.

O valor limite de $135 / 85 \mathrm{mmHg}$ tem sido recomendado por organismos internacionais, como o JNC-VI, e nacionais, como o III Consenso Brasileiro de Hipertensão Arterial ${ }^{66}$. Em situações especiais, como no diabete melito, em crianças e gestantes ainda não estão estabelecidos os critérios de normalidade.

\section{Interpretação dos dados obtidos e produção de relatórios}

Descrever o protocolo utilizado - Como o protocolo depende do objetivo do exame, a sua descrição é fundamental. 
Qualidade do procedimento - O registro deverá ser aceito para interpretação quando atingir, pelo menos, $80 \%$ de leituras válidas em relação às leituras previstas. Deverão ser excluídas as medidas aberrantes, tais como diastólica acima de $140 \mathrm{mmHg}$ e abaixo de $40 \mathrm{mmHg}$, sistólica abaixo de $70 \mathrm{mmHg}$ e acima de $250 \mathrm{mmHg}$ e pressão de pulso menor que $20 \mathrm{mmHg}$.

Médias de pressãa - De acordo com o banco de dados internacional, as médias de pressão são consideradas normais quando iguais ou menores do que $135 / 85 \mathrm{mmHg}$. Recomendamos considerar exame anormal quando as médias estiverem acima de 135/85 mmHg para registros de, no mínimo, três dias, desprezando-se o primeiro dia. No relatório deve-se citar, além das médias diárias e total do registro, a ocorrência de medidas mais elevadas no primeiro dia de registro, que podem estar relacionadas a reação de alarme, bem como medidas de outros dias que contrastem com a média de todas as medidas. Tambémé interessante analisar a média dos valores de pressão dos períodos da manhã e noite, principalmente em pacientes sob terapêutica medicamentosa.

Correlação com sintomas e medicação - Devem ser descritos no relatório de acordo com as anotações do diário do paciente. Assim, a medida residencial surge como mais uma ferramenta que pode auxiliar no manejo do paciente hipertenso, facilitando não só a identificação da real elevação dos níveis tensionais como também melhor avaliando a efetividade do tratamento instituído e favorecendo uma melhor adesão ao tratamento.

\section{Referências}

1. Clark LA, Denby L, Pregibon D, et al. Effects of activity on diurnal variations of blood pressure. J Chron Dis, 1987; 40: 671-81.

2. Mauck GB, Smith CR, Geddes LR, et al. The meaning of the point of maximum oscillations in cuff pressure in the indirect measurement of blood pressure II. J Biomech Eng 1980; 102: 28-33.

3. O'Brien E, Coats A, Owens P, et al. Use and interpretation of ambulatory blood pressure monitoring: recommendations of the British Hypertension Society. $\mathrm{Br}$ Med J 2000; 320: 1128-34.

4. Chaves JR HC. Monitorização Ambulatorial da Pressão Arterial (MAPA-24h): sua reprodutibilidade e importância na construção de modelos para estimar a hipertrofia cardíaca (tese de doutorado). Faculdade de Medicina da USP, São Paulo, 2000.

5. Omboni S, Parati G, Palatini P, et al. Reproducibility and clinical value of nocturnal hypotension: prospective evidence from the SAMPLE study. J Hypertens 1998; 16: 733-88.

6. Mochizuki Y, Okutani M, Donfeng Y, et al. Limited reproducibility of circadian variation in blood pressure dippers and non-dippers. Am J Hypertens 1998; 11(Pt 1): 403-09.

7. Ramsay LE, Williams B, Johnston GD, Macgregor GA, Poston L, Potter JF. Guidelines for management of hypertension: report of the third working party of the British Hypertension Society. J Hum Hypertens 1999; 13: 569-92.

8. Lurbe E, Aguilar F, Gomez A, Tacons J, Alvarez V, Redon J. Reproducibility of ambulatory blood pressure monitoring in children. J Hypertens 1993; 11(suppl 5): S288-S9.

9. Belsha CW, Spencer III HJ, Berry PL, Plummer JK, Wells TG. Diurnal blood pressure patterns in normotensive and hypertensive children and adolescents. J Hum Hypert 1997; 11: 801-06.

10. Sochett EB, Poon I, Balfe W, Daneman D. Ambulatory blood pressure monitoring in insulin dependent diabetes mellitus adolescents with and without microalbuminuria. J Diab Complications 1998; 12: 18-23.

11. Seeman T, Sikut M, Konrad M, Vondrichova H, Janda J, Scharer K. Blood pressure and renal function in autosomal dominant polycystic kidney disease. Pediatr Nephrol 1997; 11: 592-6.

12. Lingens N, Soergel M, Chantal L, Busch C, Lemmer B, Schörer K. Ambulatory blood pressure monitoring in paediatric patients treated by regular haemodialysis and peritoneal dialysis. Pediatr Nephrol 1995; 9: 167-72.

13. Koch VH, Furusawa E, Ignez E, Okay Y, Mion Jr D. Ambulatory blood pressure monitoring in chronically dialysed pediatric patients. Blood Pressure Monit 1999; 4: 213-16.

14. Calzolari A, Giordano U, Matteucci MC, et al. Hypertension in young patients after renal transplantation: ambulatory blood pressure monitoring versus casual blood pressure. Am J Hypert 1998; 11: 497-501.

15. Contard S, Chanudet X, Coisne D, et al. Ambulatory monitoring of blood pressure in normal pregnancy. Am J Hypertens 1993; 6: 880-4

16. Brown MA, Robinson A, Bowyer L, et al. Ambulatory blood pressure monitoring in pregnancy: what is normal? Am J Obstet Gynecol 1998; 178:. 836-42.

17. Murnaghan GA, Mitchell RH, Ruff S. Circadian variation of blood pressure in pregnancy. In: Bonnar J, MacGillivray I, Symonds EM (eds.). Pregnancy hypertension. Lancaster: MTP Press, 1980: 107-12.

18. Clark S, Hofmeyr GJ, Coats A. Ambulatory blood pressure monitoring during pregnancy: validation of the TM 2420 monitor. Obstet Gynecol 1991; 77: 152-5.

19. Halligan A, O'Brien E, O'Malley K. Twenty four hour ambulatory blood pressure measurement in a primigravid population. J Hypert 1993; 11: 869-73.

20. Peek M, Shennan A, Halligan A, Lambert PC, Taylor DJ, De Swiet M. Hypertension in pregnancy: which method of blood pressure measurement is most predictive of outcome? Obstet Gynecol 1996; 88: 1030-3.

21. Hermida RC, Ayala DE. Diagnosing gestational hypertension and preeclampsia with the 24-hour mean of blood pressure. Hypertension 1997; 30: 1531-7.

22. Olofsson P, Persson K. A comparison between conventional and 24 - hour automatic blood pressure monitoring in hypertensive pregnancy. Acta Obstet Gynecol Scand 1995; 74: 429-33.

23. Luders C, Mion JrD, Kahhale S, Zugaib M, Sabbaga E, Marcondes M. Nocturnal blood pressure fall in pre-eclamptic patients, confirmed by renal biopsy. Am J Hypertens 1994; 7(4 pt 2): 113A.

24. Pickering TG, James GD, Boddie C, Harshfield GA, Blank S, Laragh JH. How common is white coat hypertension? JAMA 1988; 259: 225-8.

25. Liu JE, Roman MJ, Pini R, Schwartz JE, Pickering TG, Devereux RB. Cardiac and arterial target organ damage in adults with elevated ambulatory and normal office blood pressure. Ann Intern Med 1999; 131: 564-72.

26. Julius S, Mejia A, Jones K, Krause L, Schork N, Van de Vem C. "White coat" versus "sustained" borderline hypertension in Tecumseh, Michigan. Hypertension 1990; 16: 617-23.

27. Cavallini MC, Roman MJ, Pickering TG, Schwartz JE, Pini R, Devereux RB. Is white coat hypertension associated with arterial disease or left ventricular hypertrophy? Hypertension 1995; 26: 413-19.

28. Ohkubo T, Hozawa A, Nagai K, et al. Prediction of stroke by ambulatory blood pressure monitoring versus screening blood pressure measurements in a general population: the Ohasama study. J Hypertens 2000; 18: 847-54.

29. Schillaci G, Verdecchia P, Sacchi N, et al. Clinical relevance of office underestimation of usual blood pressure in treated hypertension. Am J Hypertens 2000; 13(5 pt 1): 523-8.

30. Thijs L, Staessen J, Fagard R, Zacariah P, Amery A. Number of measurements required for the analysis of diurnal blood pressure profile. J Human Hypertens 1994; 8: 239-44.

31. O' Brien, Sheridan J, O' Malley K. Dippers and non-dippers. Lancet 1988; 13: 397.

32. Kario K, Matsuo T, Kobayashi H, Imiya M, Matsuo M, Shimada K. Nocturnal fall of blood pressure and silent cerebrovascular damage in elderly hypertensive patients. Advanced silent damage in extrure dippers. Hypertension 1996; 27: 130-5.

33. Mion Jr D, Nobre F. Manual de instalação e cuidados. Lemos Editorial, 1998.

34. Zacariah PK, Sheps SG, Ilstrip DM, et al. Blood Pressure: a better determinant of hypertension. Mayo Clin Proc 1988; 63: 1085-91.

35. White WB. Blood Pressure load and target organ effects in patients with essential hypertension. J Hypertens 1991; 9(suppl 8): S39-S41

36. Staessen J, Fagard R, Thijs L, Amery A, et al. A consensus view on the technique of Ambulatory Blood Pressure Monitoring. Hypertension 1995; 26(part 1): 912-18.

37. Consensus on non-invasive Ambulatory Blood Pressure Monitoring. J Hypertens 1990; 8(suppl 6): 135-40.

38. Myers MG, Brian R, Rabki. Canadian Hypertension Society Guidelines for Ambulatory Blood Pressure Monitoring. Am J Hypertens 1999; 12: 1149-57.

39. Nobre F. Análise dos dados obtidos e emissão de laudos. In: Mion Jr D, Nobre F, Monitorização Ambulatorial da Pressão Arterial. $2^{\text {a }}$ ed. São Paulo: Atheneu, 1998.

40. Ayman D, Goldshine AD. Blood pressure determinations by patients with essential hypertension: the difference between clinic and home readings before treatment. Am J Med Sci 1940; 200: 465-70.

41. Gomes MAM, Pierin A, Segre CA, Mion Jr D. Monitorização residencial da pressão arterial e monitorização ambulatorial da pressão arterial versus medida da pressão arterial no consultório. Arq Bras Cardiol 1998; 71: 581-5.

42. Padfield PL, Lindsay BA, MC Laren JA, et al. Changing relation between home and clinic blood pressure measurements: do home measurements predict clinic hypertension? Lancet 1997; 2: 322-4. 
43. Bortolotto LA, Henry O, Hanon O, et al. Validation of two devices for selfmeasurement of blood pressure by elderly patients according to the revised British Hypertension Society protocol: the Omron HEM-722C and HEM735C. Blood Pressure Monit 1999; 4: 21-5.

44. Friedman RH, Kazis LE, Jette A, et al. A tele communications system for monitoring and counseling patients with hypertension. Impact on medication adherence and blood pressure control. Am J Hypertens 1996; 9: 285-92.

45. Imai $\mathrm{Y}$, Poncelet $\mathrm{P}$, Debuyzere M, et al. Prognostic significance of selfmeasurements of blood pressure. Blood Press Monit 2000; 5: 137-43.

46. Yarows S. Home blood pressure monitoring in primary care. Blood Pressure Monitoring 1998; 3: S11-S17.

47. Pickering TG, Krakoff L, et al. American Society of Hypertension Expert Panel: conclusions and recommendations on the clinical use of home (self) and ambulatory blood pressure monitoring. Am J Hypertens 1996; 9: 1-11.

48. Vaur L, Dubroca I, Dutry-Aupagne C, et al. Superiority of home blood pressure measurements over office measurements for testing antihypertensive drugs. Blood Press Monit 1998; 3: 107-114.

49. Soghikian K, Casper SM, Fireman BH, et al. Home blood pressure in management of hypertension. Can Med Assoc J 1978; 119: 1034-9.

50. Staessen JA, O'Brien ET, Thijs L, Fagard RH. Modern approaches to blood pressure measurements. Occup Eviron Med 2000; 57: 510-20.

51. Campbell NRC, Abbott D, Bass M, et al. Self-measurement of blood pressure: recommendations of the Canadian Coalition for High Blood Pressure Prevention and Control. Canad J Cardiol 1995; 11: 5H-19H.

52. Mengden T, Chamontin B, Chau NP, et al. User procedure for self-measurements of blood pressure. Blood Press Monit 2000; 5: 111-29.

53. O'Brien E, Régis DG, Guillaume B, et al. Devices and validation. Blood Press Monit 2000; 5: 93-100.

54. O'Brien E. Replacing the mercury sphygmomanometer. BrMed J 2000; 320: 815-6.

55. Joint National Commitee on Detection, Evaluation and Treatment of High Blood
Pressure. The sixth report of the Joint National Committee on Detection, Evaluation and Treatment of High Blood Pressure (JNC-VI). Arch Intern Med 1997; 157: 2413-46.

56. O'Brien E, Mee F, Atkins N, et al. Evaluation of three devices for selfmeasurement of blood pressure according to revised British Hypertension Society Protocol: the Omron HEM-705 CP, Philips HP 5332, and Nissei DS175. Blood Press Monit 1996; 1: 55-61.

57. Stergiou GS, Skeva II, Zourbaki AS, et al. Self-monitoring of blood pressure at home: how many measurements are needed? J Hypertens 1998; 16: 725-31.

58. Stergiou GS, Zourbaki AS, Skeva II, et al. White coat effect detected using selfmonitoring of blood pressure at home. Am J Hypertens 1998; 11: 820-7.

59. Gomes MAM, Pierin A, Mion Jr D,et al. Home blood pressure measurement with a automatic device (OMRON IC): acceptance and comparison office blood pressure measurement. Hypertens 1999; 12(4 pt 2): 163A.

60. Gerin W,Pickering TG, Holland JK, Alter R. Telephone-linked home blood pressure monitoring may improve management. Am J Hypertens 1999; 12(4 pt 2): 163A.

61. Denolle T, Waeber B, Kjeldsen S, et al. Self-measurement of blood pressure in clinical trials and therapeutics applications. Blood Press Monit 2000; 5: 145-9.

62. Tsuji I, Imai Y, Nagai K, et al. Proposal of reference values for home blood pressure measurement. Prognostic criteria based on a prospective observation of the general population in Ohasama, Japan. Am J Hypertens 1997; 10: 409-18.

63. Julius S, Mejia A, Jones K, et al. "White coat" versus "sustained" borderline hypertension in Tecumseh, Michigan. Hypertension 1990; 16: 617-23.

64. Thijs L, Staessen JA, Celis H, et al. Reference values for self-recorded blood pressure. A meta-analysis of summary data. Arch Intern Med 1998; 158: 481-8.

65. Weisser B, Mengden T, Düssing R, et al. Normal values of blood pressure selfmeasurement in view of the 1999 World Health Organization - International Society of Hypertension Guidelines. Am J Hypertens 2000; 13: 340-3.

66. III Consenso Brasileiro de Hipertensão Arterial. III CBH. Rev Bras Clin Ter 1998; 24: 231-72. 